\title{
Whole-genome fetal and maternal DNA methylation analysis using MeDIP-NGS for the identification of differentially methylated regions
}

\author{
ANNA KERAVNOU ${ }^{1}$, MARIOS IOANNIDES ${ }^{2}$, KYRIAKOS TSANGARAS ${ }^{2}$, \\ CHARALAMBOS LOIZIDES ${ }^{2}$, MICHAEL D. HADJIDANIEL ${ }^{1}$, \\ ELISAVET A. PAPAGEORGIOU ${ }^{1,2}$, SKEVI KYRIAKOU ${ }^{1}$, PAVLOS ANTONIOU ${ }^{2}$, \\ PETROS MINA ${ }^{2}$, ACHILLEAS ACHILLEOS ${ }^{2}$, MARIA NEOFYTOU ${ }^{1}$, ELENA KYPRI ${ }^{1,2}$, \\ CAROLINA SISMANI ${ }^{3}$, GEORGE KOUMBARIS ${ }^{1,2}$ AND PHILIPPOS C. PATSALIS ${ }^{1,2 *}$ \\ ${ }_{2}^{1}$ Translational Genetics Team, The Cyprus Institute of Neurology and Genetics, Nicosia, Cyprus \\ ${ }_{3}^{2}$ NIPD Genetics, Nicosia, Cyprus \\ ${ }^{3}$ Department of Cytogenetics and Genomics, The Cyprus Institute of Neurology and Genetics, Nicosia, Cyprus
}

(Received 29 July 2016; accepted 23 August 2016)

\begin{abstract}
Summary
DNA methylation is an epigenetic marker that has been shown to vary significantly across different tissues. Taking advantage of the methylation differences between placenta-derived cell-free DNA and maternal blood, several groups employed different approaches for the discovery of fetal-specific biomarkers. The aim of this study was to analyse whole-genome fetal and maternal methylomes in order to identify and confirm the presence of differentially methylated regions (DMRs). We have initially utilized methylated DNA immunoprecipitation (MeDIP) and next-generation sequencing (NGS) to identify genome-wide DMRs between chorionic villus sampling (CVS) and female non-pregnant plasma (PL) and peripheral blood (WBF) samples. Next, using specific criteria, 331 fetal-specific DMRs were selected and confirmed in eight CVS, eight WBF and eight PL samples by combining MeDIP and in-solution targeted enrichment followed by NGS. Results showed higher enrichment in CVS samples as compared to both WBF and PL samples, confirming the distinct methylation levels between fetal and maternal DNA for the selected DMRs. We have successfully implemented a novel approach for the discovery and confirmation of a significant number of fetal-specific DMRs by combining for the first time MeDIP and in-solution targeted enrichment followed by NGS. The implementation of this double-enrichment approach is highly efficient and enables the detailed analysis of multiple DMRs by targeted NGS. Also, this is, to our knowledge, the first reported application of MeDIP on plasma samples, which leverages the implementation of our enrichment methodology in the detection of fetal abnormalities in maternal plasma.
\end{abstract}

\section{Introduction}

Chromosomal aneuploidies are the most common causes of genetic defects during the first trimester, which constitutes the major reason for pregnant women considering prenatal diagnosis (Hassold et al., 2007). Prenatal screening tests are usually performed in the first or second trimester of pregnancy by combining ultrasound findings with biochemical screening, providing a risk factor with limited

\footnotetext{
* Corresponding author: Prof. Philippos C. Patsalis, Translational Genetics Team, The Cyprus Institute of Neurology and Genetics, Nicosia, Cyprus. E-mail: p.patsalis@nipd.com
}

sensitivity and specificity. Currently, the gold standard in prenatal diagnosis is provided by invasive procedures, such as chorionic villus sampling (CVS) or amniocentesis, which are associated with a significant risk of fetal loss (Hulten et al., 2003). Thus, in the last decade, great interest has been shown towards the development of noninvasive prenatal testing (NIPT) methodologies that can be offered to all pregnant women without any risk of miscarriage.

The discovery of cellfree fetal DNA (cffDNA) in maternal plasma was pivotal in the development of NIPT (Lo et al., 1997). Recent studies have shown that fetal DNA concentrations in the maternal circulation are estimated to be approximately $10 \%$ during the 
first trimester (Lun et al., 2008). Several research groups utilized the presence of cffDNA in maternal blood and were able to develop different approaches for the identification and utilization of fetalspecific biomarkers for NIPT. Early studies focused on the identification of fetalspecific markers, such as DYS14, RhesusD and paternally inherited polymorphic loci, which are easily distinguishable in maternal circulation (Lo et al., 1998; Daniels et al., 2006). Despite efforts aimed at the discovery of fetalspecific markers that can be detected in all pregnancies irrespective of fetal gender and polymorphisms, the limited amount of cffDNA in the presence of a high maternal background presents a major challenge for the detection of fetal aneuploidies.

DNA methylation is an epigenetic marker that involves the addition of a methyl group on carbon 5 of the cytosine present in $\mathrm{CpG}$ dinucleotides. It has been shown that methylation patterns across different tissues vary significantly. As such, tissuespecific differentially methylated regions (DMRs) have been utilized as biomarkers for disease monitoring and prognosis, especially in the field of cancer research (Paulsen \& FergusonSmith, 2001; Baylin, 2005; Chan et al., 2013). In the prenatal setting, taking advantage of the methylation differences between the placentaderived cffDNA and the maternal peripheral blood DNA, several groups employed bisulphite conversion and methylationsensitive restriction digestion for the identification of fetalspecific DMRs that can potentially be used for the detection of fetal aneuploidies. These studies resulted in the discovery of only a small number of DMRs, including SERPINB5, AIRE, SIM2, ERG, U-PDE9A and RASSFIA (Old et al., 2007; Chim et al., 2008).

Methylated DNA immunoprecipitation (MeDIP) in combination with highresolution tiling oligonucleotide array analysis was first introduced in the field of NIPT in 2009 by Papageorgiou et al. for the discovery of fetalspecific DMRs. Specifically, more than 2000 DMRs were identified between placental and female nonpregnant peripheral blood (WBF) on each of chromosomes 21, 18, 13, X and Y (Papageorgiou et al., 2009). MeDIP in combination with realtime quantitative polymerase chain reaction (MeDIPqPCR) was later introduced for the quantification of selected DMRs on chromosome 21 , resulting in $100 \%$ sensitivity and specificity for the detection of trisomy 21 (Papageorgiou et al., 2011). Using the same approach, a second validation study of 175 cases yielded $100 \%$ sensitivity and $99.2 \%$ specificity for the NIPT of trisomy 21 (Tsaliki et al., 2012). An expansion of the fetal biomarker panel was also introduced by our group, providing the first step towards the development of NIPT assays for trisomy 18 (Ioannides et al., 2014).

Recently, the advent of nextgeneration sequencing (NGS) has revolutionized the development of NIPT
(Fan et al., 2012; Kitzman et al., 2012), providing new opportunities for the detection of fetal aneuploidies (Chiu et al., 2008; Lo et al., 2010) and other genetic abnormalities (Chen et al., 2013). In addition, methylated DNA enrichment methods and bisulphite conversion followed by NGS have been utilized to investigate the fetal methylome and its potential use in the development of methylationbased NIPT (Papageorgiou et al., 2009; Lun et al., 2013; Papageorgiou et al., 2014).

In the present study, we have utilized MeDIP in combination with NGS for genomewide fetalspecific DMR identification in CVS, wholeblood nonpregnant female samples (WBF) and female nonpregnant plasma (PL) samples. Using a novel doubleenrichment approach (MeDIP in combination with insolution hybridization enrichment followed by NGS), we have confirmed the presence of a set of 331 DMRs in multiple CVS, WBF and PL samples. The results of this study demonstrate that there is a clear distinction between the methylation levels of fetal and maternal DNA for the selected DMRs. The utilization of a novel doubleenrichment approach in this study provides a significant expansion in the number of fetalspecific biomarkers. This increase in fetal biomarkers sets the foreground for the implementation of our approach in the detection of the most common fetal aneuploidies.

\section{Materials and methods}

\subsection{Sample collection and DNA extraction}

In total, $11 \mathrm{WBF}, 10 \mathrm{PL}$ and 11 firsttrimester CVS (11-14 weeks of gestation) were used in this study (Table 1). The study has been approved by the Cyprus National Bioethics Committee and informed written consent was obtained from all participants. All WBF and CVS samples were collected from collaborating centres of the Translational Genetics Team and the Department of Cytogenetics and Genomics at the Cyprus Institute of Neurology and Genetics (Nicosia, Cyprus). PL samples were obtained from Sera Laboratories International Ltd (Sussex, UK).

Peripheral blood was collected from women donors into two 8-mL EDTAcontaining tubes. An average of $8 \mathrm{~mL}$ of plasma was isolated using a doublecentrifugation protocol as previously described (Huang et al., 2008).

WBF and CVS samples were used to extract DNA using the QIAamp Blood Midi Kit (Qiagen, Hilden, Germany) and the QIAamp DNA Mini kit (Qiagen), respectively, according to the manufacturer's instructions. DNA from PL samples was extracted using the QIAsymphony DSP Virus/Pathogen Mini Kit (Qiagen). 
Table 1. Number of samples used in our study

\begin{tabular}{lcccl}
\hline \hline Experimental step & CVS samples & PL samples & WBF samples & Sequencing type \\
\hline DMR discovery & 3 & 2 & 3 & Wholegenome sequencing \\
DMR confirmation & 8 & 8 & 8 & 331 target capture probes \\
Total & 11 & 10 & 11 & \\
\hline \hline
\end{tabular}

CVS: chorionic villus sampling; PL: female nonpregnant plasma sample; WBF: female nonpregnant whole blood.

\subsection{Experimental design}

DMR identification was initially performed on three WBF, three CVS and two PL samples using wholegenome MeDIPNGS. Based on specific criteria (see Section 3.2) we selected 331 DMRs that were found to be hypermethylated in the fetal tissues and hypomethylated in maternal whole blood and plasma. Confirmation of the methylation status of the 331 DMRs was performed on eight CVS, eight WBF and eight PL samples using MeDIP followed by targeted insolution enrichment and NGS.

\subsection{MeDIP and sequencing library construction}

Extracted DNA was quantified by realtime qPCR using the DYS14 and $\beta$ globin loci, as described previously (Zimmermann et al., 2005). Genomic DNA ranging from $18 \mathrm{ng}-3 \mu \mathrm{g}$ was sheared to an average size of $230 \mathrm{bp}$ using the Bioruptor Twin Sonicator (UCD400, Diagenode, Liege, Belgium). For DMR discovery, bluntending and sequencingadaptor ligation were performed prior to MeDIP using NEB Blunting and Ligase enzymes (NEB, Ipswich, UK), as previously described (Meyer \& Kircher, 2010; Tsangaras et al., 2014; Koumbaris et al., 2016). For DMR confirmation, bluntending and adaptor ligation were performed using the iDEAL Library Preparation kit (Diagenode), following the manufacturer's protocol.

For DMR discovery, MeDIP was performed on three CVS and three WBF samples using the MagMeDIP kit (Diagenode), according to the manufacturer's protocol. The remaining MeDIP experiments (10 PL, 8 CVS and $8 \mathrm{WBF}$ ) were performed using mouse anti5'methylcytosine monoclonal antibody (Eurogentec, Saraing, Belgium), as described previously using 18-30 ng of genomic DNA (Borgel et al., 2012). In summary, hypermethylated DNA was captured for 2 hours at $4{ }^{\circ} \mathrm{C}$ using $3 \mu \mathrm{L}$ of Dynabeads M-280 sheep antimouse IgG magnetic beads (Life Technologies, Carlsbad, CA, USA) and was washed three times with $1700 \mu \mathrm{l}$ of $1 \times$ immunoprecipitation buffer. The captured DNA was subsequently released using $1.4 \mu \mathrm{l}$ of proteinase $\mathrm{K}$ (Roche, Mannheim, Germany) by heating at $50{ }^{\circ} \mathrm{C}$ for 30 minutes. Three technical replicates were performed for each sample, which were then pooled prior to the cleanup step. All cleanup reactions were performed using the QIAquick PCR Purification Kit (Qiagen), following the manufacturer's instructions.

After MeDIP, sequencing libraries used for DMR discovery were amplified for 15 cycles as described previously (Meyer \& Kircher, 2010; Koumbaris et al., 2016). After MeDIP, these libraries, which were used for the confirmation of selected DMRs, were amplified using the iDEAL Library Preparation Kit (Diagenode), following the manufacturer's protocol.

\subsection{Design and preparation of target capture probes}

OligoAnalyzer $3 \cdot 1$ software was used to design specific primers in order to generate $144-160 \mathrm{bp}$ long target capture probes for each of the selected DMRs (Owczarzy et al., 2008). PrimerBLAST (NCBI) and in silico PCR (UCSC Genome browser) were used in order to confirm primer specificity. PCR reactions were performed using MyTaq HS DNA Polymerase (BioLine, London, UK), as described elsewhere (Koumbaris et al., 2016). Capture probes were confirmed by agarose gel electrophoresis and were purified using the QIAquick PCR Purification Kit. The concentration of each probe was measured using the NanoDrop spectrophotometer (Thermo Scientific, Wilmington, MA USA) and probes were pooled equimolarly. Pooled probes were biotinylated and immobilized on streptavidincoated magnetic beads (Thermo Scientific, Vilnius, Lithuania), as previously described (Tsangaras et al., 2014).

\subsection{Targeted enrichment and sequencing}

For targeted enrichment, $700-1200 \mathrm{ng}$ of each barcoded library was mixed with $2 \times$ hybridization buffer (Agilent, Santa Clara, CA, USA), $10 \times$ blocking agent (Agilent), blocking oligonucleotides (Maricic et al., 2010), human Cot1 (Invitrogen, Carlsbad, CA, USA) and salmon sperm DNA (Invitrogen) (Maricic et al., 2010; Koumbaris et al., 2016). Immunoprecipitated libraries were incubated with the biotinylated capture probes for 48 hours at $66^{\circ} \mathrm{C}$ and were eluted by heating, as previously described (Tsangaras et al., 2014). Enriched samples were amplified using 
outwardbound adaptor primers (Tsangaras et al., 2014) and were quantified using the KAPA Library Quantification KitIllumina (KAPA Biosystems, Boston, MA, USA). The enriched barcoded libraries were then pooled equimolarly and were subjected to pairedend sequencing on an Illumina HiSeq 2500 system (Illumina, San Diego, CA, USA).

\subsection{Read trimming and sequence alignment}

Sequencing reads were trimmed with cutadapt v.1.2 (Martin, 2011), normalized and aligned to the human reference genome GRCh37/hg19 using the BWA v.0.7.4 MEM algorithm (Li \& Durbin, 2009). The Picard tool was used to remove duplicate reads and to convert aligned reads to a binary (BAM) file. Only the uniquely aligned and highquality (quality score $>30$ ) reads were used in the sequencing analysis. Local realignment and base recalibration was performed with GATK (McKenna et al., 2010). The SAMtools software was used to retrieve the read depth of each base (Li et al., 2009).

\subsection{Data analysis}

\subsubsection{DMR discovery}

Candidate fetalspecific DMRs were selected according to the following criteria: (a) the regions selected exhibited consistent DNA hypermethylation profiles in all CVS and hypomethylation in all the female nonpregnant tissues; (b) selected regions had preferentially more than two $\mathrm{CpG}$ dinucleotides in the DNA sequence; (c) potential DMRs that were in copy number variable regions or in repetitive element regions were excluded; (d) selected DMRs should be located 200 bp away from repetitive elements; and (e) the adjusted (Bonferroni correction) $P$ value of the window bins that covered the region and were obtained from the MEDIPS test for methylation differences was less than $0 \cdot 10$.

Pairwise genomewide methylation comparisons between CVS, WBF and PL were performed using the MEDIPS package (Chavez et al., 2010) with window sizes (bins) specified at $100 \mathrm{bp}$. Window bins with adjusted $P$ value $<0.1$ were considered for subsequent analysis. These bins were merged into consecutive regions (DMRs) after specific criteria selection and filtering.

\subsubsection{DMR confirmation}

Prior to analyses, all sequenced reads were normalized by employing 'vertical normalization'. This normalization method equalizes the cumulative read depth of the selected DMRs across all samples.
A mixedeffects analysis of variance model was applied in order to compare the overall methylation levels of the three tissue types (CVS, WBF and PL). The response variable in this model is the read depth, which in our experiments acts as a proxy for the methylation level of each region, while the categorical variable is the sample type and consists of three levels (CVS, WBF and PL). The additional random effect allows for different methylation variability between the different DMRs. Subsequent posthoc pairwise comparisons were applied in order to identify the exact nature of the differences between the three tissues. Three pairwise Welch's t tests were applied and the resulting $P$ values were corrected using the Holm-Bonferroni method (Holm, 1979).

\section{Results}

\subsection{DMR discovery using wholegenome DNA} methylation analysis of $W B F, C V S$ and PL samples

Three WBF, three CVS and two PL samples were subjected to wholegenome MeDIPNGS analysis to enable genomewide identification of fetalspecific DMRs. The resulting alignment files were used as input for the R package MEDIPS, where the differential coverage between two groups of samples (i.e. CVS vs. WBF and CVS vs. PL) was calculated. Using MEDIPS criteria (see Section 2.7.1), 3574 DMRs were identified in the CVS vs. WBF comparisons, of which 1888 regions showed hypermethylation in CVS and hypomethylation in WBF samples, while 1686 regions showed hypomethylation in CVS and hypermethylation in WBF samples (Table 2). Similarly, 8091 DMRs were identified in the CVS vs. PL comparison, of which 6313 were hypermethylated in CVS and hypomethylated in PL samples, whereas 1778 regions were hypomethylated in CVS and hypermethylated in PL samples (Table 3). Identified DMRs were distributed across all autosomes. Regions on X- and Y-chromosomes were excluded from the analysis.

\subsubsection{Methylation differences between fetal and maternal tissue}

The overlap of the DMRs obtained from the two comparisons (WBF vs. CVS and PL vs. CVS) provided 1453 common fetalspecific DMRs that showed hypermethylation in CVS and hypomethylation in maternal samples. Those ranged from 100 to $2300 \mathrm{bp}$ in length. Comparison of the overall methylation status of the aforementioned DMRs showed a clear distinction of the methylation status between CVS and maternal tissues (WBF and PL), with adjusted $P<2 \times 10^{-16}$ for all three pairwise posthoc tests (Fig. 1(a)). Overall, the DNA methylation enrichment in CVS was greater 
Table 2. DMRs identified between CVS and WBF DNA samples

\begin{tabular}{|c|c|c|c|c|c|c|}
\hline \multirow[b]{2}{*}{ CHR } & \multicolumn{3}{|c|}{ No. of DMRs } & \multicolumn{3}{|c|}{$\% \mathrm{CVS}$ versus $\mathrm{WBF}$} \\
\hline & $\begin{array}{l}\text { No. of HYPER } \\
\text { in CVS }\end{array}$ & $\begin{array}{l}\text { No. of HYPO } \\
\text { in CVS }\end{array}$ & Total no. & $\begin{array}{l}\% \text { HYPER } \\
\text { in CVS }\end{array}$ & $\begin{array}{l}\% \text { HYPO } \\
\text { in CVS }\end{array}$ & $\begin{array}{l}\% \text { whole } \\
\text { genome }\end{array}$ \\
\hline chr1 & 171 & 175 & 346 & 9.06 & $10 \cdot 38$ & $9 \cdot 68$ \\
\hline chr2 & 173 & 138 & 311 & $9 \cdot 16$ & $8 \cdot 19$ & $8 \cdot 70$ \\
\hline chr3 & 95 & 72 & 167 & $5 \cdot 03$ & $4 \cdot 27$ & $4 \cdot 67$ \\
\hline chr4 & 78 & 51 & 129 & $4 \cdot 13$ & 3.02 & $3 \cdot 61$ \\
\hline $\operatorname{chr} 5$ & 122 & 104 & 226 & 6.46 & $6 \cdot 17$ & $6 \cdot 32$ \\
\hline chr6 & 139 & 139 & 278 & $7 \cdot 36$ & $8 \cdot 24$ & $7 \cdot 78$ \\
\hline chr7 & 85 & 81 & 166 & $4 \cdot 50$ & $4 \cdot 80$ & $4 \cdot 64$ \\
\hline chr8 & 108 & 72 & 180 & $5 \cdot 72$ & $4 \cdot 27$ & $5 \cdot 04$ \\
\hline chr9 & 37 & 32 & 69 & 1.96 & 1.90 & 1.93 \\
\hline $\operatorname{chr} 10$ & 114 & 122 & 236 & $6 \cdot 04$ & $7 \cdot 24$ & $6 \cdot 60$ \\
\hline $\operatorname{chr} 11$ & 79 & 92 & 171 & $4 \cdot 18$ & 5.46 & $4 \cdot 78$ \\
\hline $\operatorname{chr} 12$ & 112 & 69 & 181 & 5.93 & 4.09 & 5.06 \\
\hline chr13 & 62 & 76 & 138 & $3 \cdot 28$ & $4 \cdot 51$ & 3.86 \\
\hline chr14 & 97 & 60 & 157 & $5 \cdot 14$ & 3.56 & $4 \cdot 39$ \\
\hline chr15 & 46 & 59 & 105 & 2.44 & $3 \cdot 50$ & 2.94 \\
\hline chr16 & 62 & 50 & 112 & 3.28 & 2.97 & $3 \cdot 13$ \\
\hline chr17 & 93 & 71 & 164 & 4.93 & 4.21 & 4.59 \\
\hline chr18 & 37 & 45 & 82 & 1.96 & 2.67 & 2.29 \\
\hline chr19 & 39 & 60 & 99 & 2.07 & 3.56 & 2.77 \\
\hline $\operatorname{chr} 20$ & 72 & 58 & 130 & $3 \cdot 81$ & 3.44 & 3.64 \\
\hline $\operatorname{chr} 21$ & 34 & 31 & 65 & 1.80 & 1.84 & 1.82 \\
\hline chr22 & 33 & 29 & 62 & 1.75 & 1.72 & 1.73 \\
\hline Total & 1888 & 1686 & 3574 & & & \\
\hline
\end{tabular}

CHR: chromosome; CVS: chorionic villus sampling; DMR: differentially methylated region; HYPER: hypermethylated; HYPO: hypomethylated; WBF: female nonpregnant whole blood.

compared to WBF and PL, while the DNA methylation differences were less pronounced but still statistically significant between CVS and PL than between CVS and WBF.

\subsection{DMR selection}

To further ascertain and characterize the identified biomarkers, a subset of 331 potential fetalspecific DMRs (median length: $149 \mathrm{bp}$; IQR: $11 \mathrm{bp}$ ) was selected based on specific criteria (see Section 2.7.1). In addition, selection was focused on autosomal chromosomes and on regions located in significant regulatory regions such as potential promoters, $\mathrm{CpG}$ islands (CGIs) and exonic (coding) regions. Specifically, 294, 64 and 73 DMRs were located within coding regions (67.7\% in gene bodies and $32.3 \%$ in exons), potential promoters and CGIs, respectively (Table 4 \& Table S1).

\subsection{DMR confirmation}

The methylation status of the 331 DMRs was ascertained in a cohort of eight normal CVS, eight WBF and eight PL samples, using MeDIP in combination with insolution targeted enrichment followed by NGS. Overall, methylation comparisons demonstrated significant methylation differences between the three sample types for the 331 selected DMRs (Fig. 1(b)). Results showed higher enrichment in CVS samples compared to both WBF and PL samples. Furthermore, a more pronounced methylation difference was apparent between CVS and WBF than between CVS and PL.

The methylation status of the selected DMRs was also compared with previous studies that utilized methylation differences between fetal and maternal tissue for the identification of fetalspecific biomarkers. Common DMRs were found between our approach and DMRs identified using bisulphite conversion, methylationsensitive restriction digestion and microarrays (Old et al., 2007; Chim et al., 2008; Chu et al., 2009; Bunce et al., 2012; Yin et al., 2012; Lun et al., 2013; Hatt et al., 2015). More concordant results were observed between our study and DMRs identified using MeDIP approaches (Papageorgiou et al., 2009; Ioannides et al., 2014; Xiang et al., 2014).

\section{Discussion}

In this study, we undertook the genomewide biomarker discovery of DMRs between fetal and maternal DNA and confirmed the presence of a subset of 
Table 3. DMRs identified between CVS and PL DNA samples

\begin{tabular}{|c|c|c|c|c|c|c|}
\hline \multirow[b]{2}{*}{$\mathrm{CHR}$} & \multicolumn{3}{|c|}{ No. of DMRs } & \multicolumn{3}{|c|}{$\% \mathrm{CVS}$ versus $\mathrm{PL}$} \\
\hline & $\begin{array}{l}\text { No. of HYPER } \\
\text { in CVS }\end{array}$ & $\begin{array}{l}\text { No. of HYPO } \\
\text { in CVS }\end{array}$ & Total no. & $\begin{array}{l}\% \text { HYPER } \\
\text { in CVS }\end{array}$ & $\begin{array}{l}\% \text { HYPO } \\
\text { in CVS }\end{array}$ & $\begin{array}{l}\% \text { whole } \\
\text { genome }\end{array}$ \\
\hline chr1 & 587 & 177 & 764 & $9 \cdot 30$ & 9.96 & 9.44 \\
\hline $\operatorname{chr} 2$ & 528 & 163 & 691 & $8 \cdot 36$ & $9 \cdot 17$ & $8 \cdot 54$ \\
\hline $\operatorname{chr} 3$ & 310 & 99 & 409 & 4.91 & $5 \cdot 57$ & 5.05 \\
\hline chr4 & 200 & 65 & 265 & $3 \cdot 17$ & $3 \cdot 66$ & $3 \cdot 28$ \\
\hline $\operatorname{chr} 5$ & 286 & 116 & 402 & 4.53 & $6 \cdot 52$ & 4.97 \\
\hline chr6 & 412 & 153 & 565 & $6 \cdot 53$ & $8 \cdot 61$ & $6 \cdot 98$ \\
\hline $\operatorname{chr} 7$ & 311 & 66 & 377 & 4.93 & $3 \cdot 71$ & $4 \cdot 66$ \\
\hline chr8 & 238 & 90 & 328 & 3.77 & $5 \cdot 06$ & 4.05 \\
\hline $\operatorname{chr} 9$ & 146 & 34 & 180 & $2 \cdot 31$ & 1.91 & $2 \cdot 22$ \\
\hline $\operatorname{chr} 10$ & 368 & 95 & 463 & $5 \cdot 83$ & $5 \cdot 34$ & $5 \cdot 72$ \\
\hline chr11 & 204 & 123 & 327 & $3 \cdot 23$ & $6 \cdot 92$ & $4 \cdot 04$ \\
\hline $\operatorname{chr} 12$ & 366 & 105 & 471 & $5 \cdot 80$ & $5 \cdot 91$ & $5 \cdot 82$ \\
\hline chr13 & 168 & 79 & 247 & $2 \cdot 66$ & $4 \cdot 44$ & 3.05 \\
\hline chr14 & 300 & 80 & 380 & $4 \cdot 75$ & $4 \cdot 50$ & $4 \cdot 70$ \\
\hline chr15 & 282 & 84 & 366 & 4.47 & $4 \cdot 72$ & 4.52 \\
\hline $\operatorname{chr} 16$ & 237 & 34 & 271 & 3.75 & 1.91 & $3 \cdot 35$ \\
\hline $\operatorname{chr} 17$ & 378 & 33 & 411 & 5.99 & 1.86 & 5.08 \\
\hline chr18 & 132 & 70 & 202 & $2 \cdot 09$ & 3.94 & $2 \cdot 50$ \\
\hline chr19 & 215 & 21 & 236 & $3 \cdot 41$ & $1 \cdot 18$ & 2.92 \\
\hline $\operatorname{chr} 20$ & 295 & 55 & 350 & 4.67 & 3.09 & $4 \cdot 33$ \\
\hline $\operatorname{chr} 21$ & 160 & 26 & 186 & 2.53 & 1.46 & $2 \cdot 30$ \\
\hline chr22 & 190 & 10 & 200 & 3.01 & 0.56 & $2 \cdot 47$ \\
\hline Total & 6313 & 1778 & 8091 & & & \\
\hline
\end{tabular}

CHR: chromosome; CVS: chorionic villus sampling; DMR: differentially methylated region; HYPER: hypermethylated; HYPO: hypomethylated; PL: female nonpregnant plasma sample.
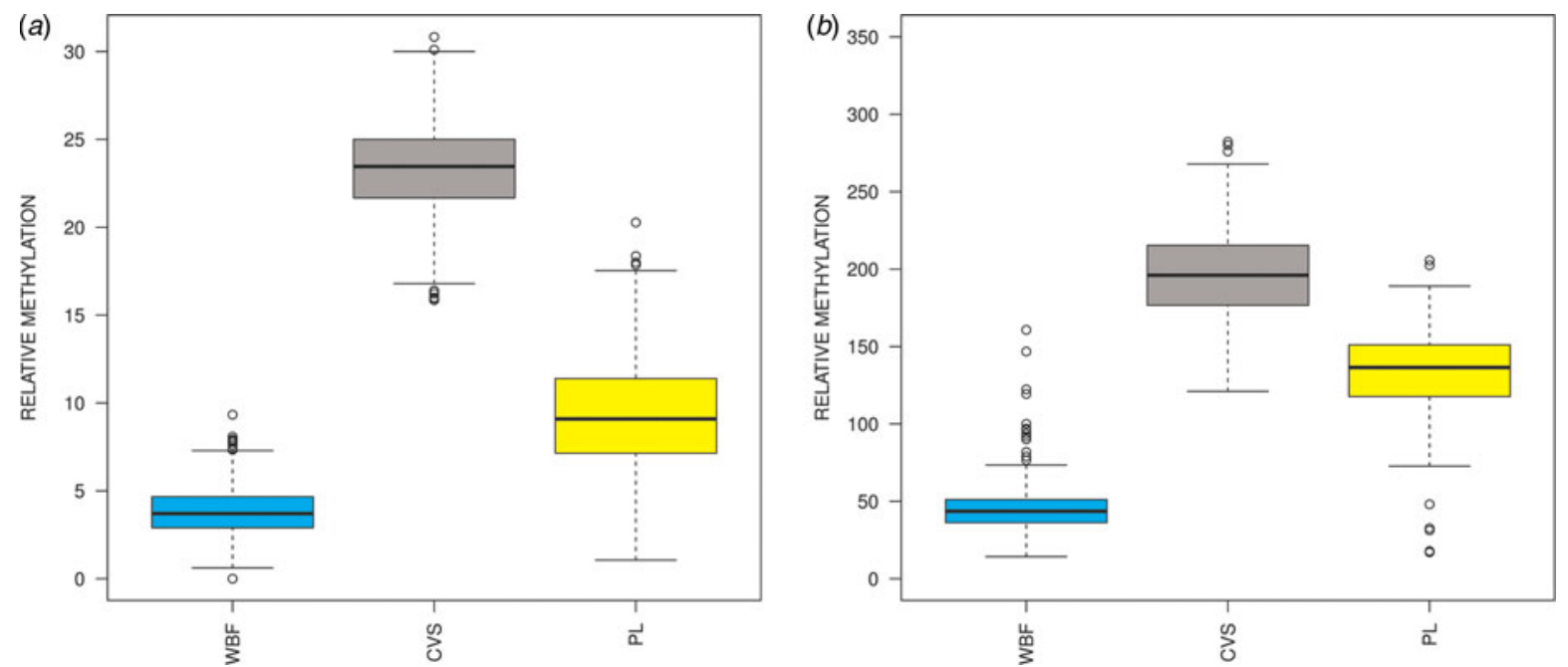

Fig. 1. DMR methylation enrichment in CVS, WBF and PL samples. Overall, DMR methylation enrichment in CVS, WBF and PL samples using $(a)$ the 1453 common fetalspecific DMRs following MeDIPNGS analysis and $(b)$ a subset of 331 fetalspecific DMRs using MeDIP in combination with insolution targeted enrichment followed by NGS. Posthoc comparisons showed significant statistical differences between the three tissues $\left(P<2 \times 10^{-16}\right)$ with higher enrichment in CVS compared to maternal DNA, confirming the hypermethylation status of the fetal DNA (CVS) as compared to the maternal DNA (WBF and PL). CVS: chorionic villus sampling; PL: female nonpregnant plasma sample; WBF: female nonpregnant whole blood.

these DMRs by combining for the first time MeDIP with insolution targeted enrichment and NGS.

Also, this is, to our knowledge, the first reported application of MeDIP in plasma samples. Previous studies have employed MeDIP in order to characterize the methylation status of different tissues using large amounts of input DNA. We were able to overcome this limitation and successfully enrich and characterize 
Table 4. Location characteristics of 331 selected DMRs

\begin{tabular}{|c|c|c|c|c|c|}
\hline Chromosome & $\begin{array}{l}\text { No. } \\
\text { DMRs } \\
\text { selected }\end{array}$ & $\begin{array}{l}\text { Gene } \\
\text { bodies }\end{array}$ & Exons & $\begin{array}{l}\text { Potential } \\
\text { promoters }\end{array}$ & CGIs \\
\hline chr1 & 34 & 13 & 6 & 9 & 12 \\
\hline $\operatorname{chr} 2$ & 10 & 10 & 7 & 1 & 3 \\
\hline $\operatorname{chr} 3$ & 10 & 7 & 5 & 1 & 4 \\
\hline $\operatorname{chr} 4$ & 12 & 7 & 5 & 2 & 4 \\
\hline $\operatorname{chr} 5$ & 12 & 8 & 4 & 1 & 5 \\
\hline chr6 & 13 & 7 & 6 & 3 & 3 \\
\hline chr7 & 28 & 16 & 5 & 7 & 5 \\
\hline chr8 & 10 & 9 & 6 & 1 & 4 \\
\hline $\operatorname{chr} 9$ & 9 & 8 & 4 & 1 & 0 \\
\hline chr10 & 15 & 12 & 5 & 2 & 0 \\
\hline chr11 & 10 & 8 & 4 & 3 & 4 \\
\hline chr12 & 12 & 10 & 6 & 2 & 2 \\
\hline chr13 & 28 & 15 & 3 & 10 & 7 \\
\hline chr14 & 11 & 4 & 1 & 3 & 2 \\
\hline $\operatorname{chr} 15$ & 9 & 7 & 4 & 1 & 1 \\
\hline chr16 & 7 & 6 & 2 & 0 & 2 \\
\hline $\operatorname{chr} 17$ & 10 & 9 & 2 & 1 & 0 \\
\hline $\operatorname{chr} 18$ & 31 & 13 & 7 & 3 & 8 \\
\hline chr19 & 6 & 3 & 1 & 2 & 0 \\
\hline chr20 & 9 & 5 & 3 & 2 & 3 \\
\hline chr21 & 39 & 18 & 8 & 9 & 4 \\
\hline $\operatorname{chr} 22$ & 6 & 4 & 1 & 0 & 0 \\
\hline Total & 331 & 199 & 95 & 64 & 73 \\
\hline
\end{tabular}

CGI: CpG island; DMR: differentially methylated region.

the methylome of multiple plasma samples using modifications of an existing MeDIP protocol (Borgel et al., 2012). As a result, we developed a MeDIPNGS methodology that allowed us to use input concentrations of as low as $18-30 \mathrm{ng}$ of plasma DNA derived from two 8-mL peripheral blood aliquots. This development constitutes another milestone in the development of an affordable epigenetic NIPT assay for the detection of fetal abnormalities in maternal plasma.

Previous studies have employed different methods for the discovery of fetalspecific biomarkers using methylation differences between fetal and maternal DNA, including sodium bisulphite conversion, methylationsensitive restriction digestion or affinitybased techniques (Gitan et al., 2002; Chim et al., 2005; Old et al., 2007; Chim et al., 2008; Laird, 2010; Tong et al., 2010). Hypermethylated fetalspecific DMRs have been the focus of different studies due to their potential to be highly enriched and readily distinguished from the maternal background, and thus to be utilized for the detection of fetal aneuploidies (Chu et al., 2009; Papageorgiou et al., 2011; Tsaliki et al., 2012; Yin et al., 2012; Xiang et al., 2014). In this study, we first undertook fetalspecific biomarker discovery between CVS, WBF and PL using MeDIPNGS (Fig. 1(a)). Subsequently, 331 DMRs (Table $4 \&$ Table S1) were selected and subjected to
MeDIP followed by targeted enrichment of eight CVS, eight WBF and eight PL samples (Fig. 1(b)). Pairwise comparisons on the three tissues based on their methylation levels confirmed that the overall methylation status of WBF and PL was significantly lower for the tested DMRs compared to the CVS samples. Further characterization of selected DMRs can therefore be performed on CVS and female plasma samples, since cffDNA is present in higher amounts in maternal plasma than in peripheral blood (Lo et al., 1998).

Comparison of the selected DMRs with previous reports showed that the methylation patterns of several DMRs that were confirmed in our study are consistent with other methylationbased approaches (Old et al., 2007; Chim et al., 2008; Chu et al., 2009; Bunce et al., 2012; Yin et al., 2012; Lun et al., 2013; Hatt et al., 2015), demonstrating that our novel, first reported, doubleenrichment approach is a robust method for the efficient and comprehensive characterization of selected DMRs. Further validation experiments are necessary in order to investigate the methylation variability in a larger cohort. It is also notable that our results show higher concordance with the results of other MeDIPbased approaches, reaffirming the reproducible nature of the MeDIP methodology (Papageorgiou et al., 2009; Tsaliki et al., 2012; Ioannides et al., 2014; Xiang et al., 2014).

Based on the characteristics of the validated DMRs and due to the great potential of this approach to be utilized in the clinical setting for the detection of the most common aneuploidies, future work will focus on the identification and characterization of additional DMRs on chromosomes 13, 18 and 21. In addition, the discovery of DMRs across all autosomes using our approach opens the way for identifying and validating markers associated with subchromosomal copy number changes, such us clinically relevant microdeletions and microduplications.

This work was supported by the 7th Framework Programme, European Research Council, for the project 'A Novel NonInvasive Prenatal Diagnosis of Genetic Disorders' (grant number 322953) and the Cyprus Institute of Neurology and Genetics (http://www.cing.ac.cy). Parts of the data analysis were carried out on the CyTera HPC Facility that is supported by the CyTera Project (NEA

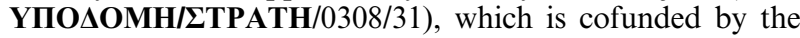
European Regional Development Fund and the Republic of Cyprus through the Research Promotion Foundation.

\section{Declaration of interest}

Some of the authors have competing interests to declare. Specifically, MI, KT, CL, EAP, PM, AA, EK, GK and PCP are employed by NIPD Genetics. Also, KT, EAP, PM, AA, EK, GK and PCP own shares in the company. At the time that the study 
was carried out, PA was employed by NIPD Genetics. Currently, he has no affiliations with NIPD Genetics and he declares no competing interests. EAP and PCP have filed a PCT patent application for the MeDIP realtime qPCRbased NIPD approach (PCT Patent Application No. PCT/1B2011/000217). The authors $\mathrm{AK}, \mathrm{MDH}, \mathrm{SK}, \mathrm{MN}$ and $\mathrm{CS}$ declare that they have no competing interests.

\section{Supplementary material}

For supplementary material accompanying this paper visit https://doi.org/10.1017/S0016672316000136.

\section{References}

Baylin, S. B. (2005). DNA methylation and gene silencing in cancer. Nature Clinical Practice Oncology 2, S4-S11.

Borgel, J., Guibert, S. \& Weber, M. (2012). Genomic imprinting. Methylated DNA Immunoprecipitation (MeDIP) from Low Amounts of Cells 925, 149-158.

Bunce, K., Chu, T., Surti, U., Hogge, W. A. \& Peters, D. G. (2012). Discovery of epigenetic biomarkers for the noninvasive diagnosis of fetal disease. Prenatal Diagnosis 32, 542-549.

Chan, K. C. A., Jiang, P., Chan, C. W. M., Sun, K., Wong, J., Hui, E. P. \& Lo, Y. M. D. (2013). Noninvasive detection of cancerassociated genomewide hypomethylation and copy number aberrations by plasma DNA bisulfite sequencing. Proceedings of the National Academy of Sciences of the United States of America 110, 1-8.

Chavez, L., Jozefczuk, J., Grimm, C., Timmermann, B., Lehrach, H., Herwig, R. \& Adjaye, J. (2010). Computational analysis of genomewide DNA methylation during the differentiation of human embryonic stem cells along the endodermal lineage. Genome Research 20, 1441-1450.

Chen, S., Lau, T. K., Zhang, C., Xu, C., Xu, Z., Hu, P. \& Zhang, X. (2013). A method for noninvasive detection of fetal large deletions/duplications by low coverage massively parallel sequencing. Prenatal Diagnosis 33, 584-590.

Chim, S. S. C., Jin, S., Lee, T. Y.H., Lun, F. M. F., Lee, W. S., Chan, L. Y. S. \& Lo, Y. M. D. (2008). Systematic search for placental DNAmethylation markers on chromosome 21: toward a maternal plasmabased epigenetic test for fetal trisomy 21. Clinical Chemistry 54, 500-511.

Chim, S. S. C., Tong, Y. K., Chiu, R. W. K., Lau, T. K., Leung, T. N., Chan, L. Y.S. \& Lo, Y. M. D. (2005). Detection of the placental epigenetic signature of the maspin gene in maternal plasma. Proceedings of the National Academy of Sciences of the United States of America 102, 14753-14758.

Chiu, R. W. K., Chan, K. C. A., Gao, Y., Lau, V. Y. M., Zheng, W., Leung, T. Y. \& Lo, Y. M. D. (2008). Noninvasive prenatal diagnosis of fetal chromosomal aneuploidy by massively parallel genomic sequencing of DNA in maternal plasma. Proceedings of the National Academy of Sciences of the United States of America 105, 20458-20463.

Chu, T., Burke, B., Bunce, K., Surti, U., Allen Hogge, W. \& Peters, D. G. (2009). A microarraybased approach for the identification of epigenetic biomarkers for the noninvasive diagnosis of fetal disease. Prenatal Diagnosis 29, 1020 10230.

Daniels, G., Finning, K., Martin, P. \& Summers, J. (2006). Fetal blood group genotyping: present and future. Annals of the New York Academy of Sciences 1075, 88-95.

Fan, H. C., Gu, W., Wang, J., Blumenfeld, Y. J., ElSayed, Y. Y. \& Quake, S. R. (2012). Noninvasive prenatal measurement of the fetal genome. Nature 487, 320-324.

Gitan, R. S., Shi, H., Chen, C.M., Yan, P. S. \& Huang, T.H.M. (2002). Methylationspecific oligonucleotide microarray: a new potential for highthroughput methylation analysis. Genome Research 12, 158-164.

Hassold, T., Hall, H. \& Hunt, P. (2007). The origin of human aneuploidy: where we have been, where we are going. Human Molecular Genetics 16, 203-208.

Hatt, L., Aagaard, M. M., Graakjaer, J., Bach, C., Sommer, S., Agerholm, I. E. \& Bojesen, A. (2015). Microarraybased analysis of methylation status of $\mathrm{CpGs}$ in placental DNA and maternal blood DNA - potential new epigenetic biomarkers for cell free fetal DNAbased diagnosis. PLOS ONE 10, e0128918.

Holm, S. (1979). A simple sequentially rejective multiple test procedure. Scandinavian Journal of Statistics 6, 65-70.

Huang, D. J., MergenthalerGatfield, S., Hahn, S., Holzgreve, W. \& Zhong, X. Y. (2008). Isolation of cellfree DNA from maternal plasma using manual and automated systems. Methods in Molecular Biology 444, 203-208.

Hulten, M. A., Dhanjal, S. \& Pertl, B. (2003). Rapid and simple prenatal diagnosis of common chromosome disorders: advantages and disadvantages of the molecular methods FISH and QF-PCR. Reproduction 126, 279-297.

Ioannides, M., Papageorgiou, E. A., Keravnou, A., Tsaliki, E., Spyrou, C., Hadjidaniel, M. \& Patsalis, P. C. (2014). Interindividual methylation variability in differentially methylated regions between maternal whole blood and first trimester CVS. Molecular Cytogenetics 7, 2-8.

Kitzman, J. O., Snyder, M. W., Ventura, M., Lewis, A. P., Qiu, R., Simmons, L. E. \& Shendure, J. (2012). Noninvasive wholegenome sequencing of a human fetus. Science Translational Medicine 4, 137ra76.

Koumbaris, G., Kypri, E., Tsangaras, K., Achilleos, A., Mina, P., Neofytou, M. \& Patsalis, P. C. (2016). Cellfree DNA analysis of targeted genomic regions in maternal plasma for noninvasive prenatal testing of trisomy 21 , trisomy 18, trisomy 13 and fetal gender. Clinical Chemistry 62, 848-855.

Laird, P. W. (2010). Principles and challenges of genomewide DNA methylation analysis. Nature Reviews Genetics 11, 191-203.

Li, H. \& Durbin, R. (2009). Fast and accurate short read alignment with Burrows-Wheeler transform. Bioinformatics (Oxford, England) 25, 1754-1760.

Li, H., Handsaker, B., Wysoker, A., Fennell, T., Ruan, J., Homer, N., Marth, G., Abecasis, G. \& Durbin, R.; 1000 Genome Project Data Processing Subgroup (2009). The Sequence Alignment/Map format and SAMtools. Bioinformatics 25, 2078-2079.

Lo, Y. M. D., Chan, K. C. A., Sun, H., Chen, E. Z., Jiang, P., Lun, F. M. F. \& Chiu, R. W. K. (2010). Maternal plasma DNA sequencing reveals the genomewide genetic and mutational profile of the fetus. Science Translational Medicine 2, 1-13.

Lo, Y. M. D., Corbetta, N., Chamberlain, P. F., Rai, V., Sargent, I. L. \& Redman, C. W. G. (1997). Presence of fetal DNA in maternal plasma and serum. Lancet 350, 485-487.

Lo, Y. M. D., Hjelm, N. M., Fidler, C., L.Sargent, I., Murphy, M. F., Chamberlain, P.F. \& Wainscoat, J.S. 
(1998). Prenatal diagnosis of fetal RhD status by molecular analysis of maternal plasma. The New England Journal of Medicine 339, 1734-1738.

Lo, Y. M., Tein, M.S., Lau, T. K., Haines, C. J., Leung, T. N., Poon, P. M. \& Hjelm, N. M. (1998). Quantitative analysis of fetal DNA in maternal plasma and serum: implications for noninvasive prenatal diagnosis. American Journal of Human Genetics 62, 768-775.

Lun, F. M. F., Chiu, R. W. K., Chan, K. C., Leung, T. Y., Lau, T. K. \& Lo, Y. M. (2008). Microfluidics digital PCR reveals a higher than expected fraction of fetal DNA in maternal plasma. Clinical Chemistry 54, 1664 1672.

Lun, F. M. F., Chiu, R. W. K., Sun, K., Leung, T. Y., Jiang, P., Chan, K. C. A. \& Lo, Y. M. D. (2013). Noninvasive prenatal methylomic analysis by genomewide bisulfite sequencing of maternal plasma DNA. Clinical Chemistry 59, 1583-1594.

Maricic, T., Whitten, M. \& Pääbo, S. (2010). Multiplexed DNA sequence capture of mitochondrial genomes using PCR products. PLoS ONE 5, e14004.

Martin, M. (2011). Cutadapt removes adapter sequences from highthroughput sequencing reads. EMBnet.journal 17, 10-12.

McKenna, A., Hanna, M., Banks, E., Sivachenko, A., Cibulskis, K., Kernytsky, A. \& Mark, D. A. (2010). The Genome Analysis Toolkit: A MapReduce framework for analyzing nextgeneration DNA sequencing data. Genome Research 20, 1297-1303.

Meyer, M. \& Kircher, M. (2010). Illumina sequencing library preparation for highly multiplexed target capture and sequencing. Cold Spring Harbor Protocols 5, 1-7.

Old, R. W., Crea, F., Puszyk, W. \& Hultén, M. A. (2007). Candidate epigenetic biomarkers for noninvasive prenatal diagnosis of Down syndrome. Reproductive BioMedicine Online 15, 227-235.

Owczarzy, R., Tataurov, A. V., Wu, Y., Manthey, J.A., McQuisten, K.A., Almabrazi, H. G. \& Peek, A. S. (2008). IDT SciTools: a suite for analysis and design of nucleic acid oligomers. Nucleic Acids Research 36, 163-169.

Papageorgiou, E. a, Karagrigoriou, A., Tsaliki, E., Velissariou, V., Carter, N.P. \& Patsalis, P. C. (2011).
Fetalspecific DNA methylation ratio permits noninvasive prenatal diagnosis of trisomy 21. Nature Medicine 17, 510-513.

Papageorgiou, E. A, Koumbaris, G., Kypri, E., Hadjidaniel, M. \& Patsalis, P.C. (2014). The epigenome view: an effort towards noninvasive prenatal diagnosis. Genes $\mathbf{5}$, 310-329.

Papageorgiou, E. A., Fiegler, H., Rakyan, V., Beck, S., Hulten, M., Lamnissou, K. \& Patsalis, P. C. (2009). Sites of differential DNA methylation between placenta and peripheral blood. The American Journal of Pathology 174, 1609-1618.

Paulsen, M. \& FergusonSmith, A. C. (2001). Methylation and colorectal cancer. Journal of Pathology 195, 111-134.

Tong, Y. K., Jin, S., Chiu, R. W. K., Ding, C., Chan, K. C. A., Leung, T. Y. \& Lo, Y. M. D. (2010). Noninvasive prenatal detection of trisomy 21 by an epigeneticgenetic chromosomedosage approach. Clinical Chemistry 56, 90-98.

Tsaliki, E., Papageorgiou, E. A., Spyrou, C., Koumbaris, G., Kypri, E., Kyriakou, S. \& Patsalis, P. C. (2012). MeDIP realtime qPCR of maternal peripheral blood reliably identifies trisomy 21. Prenatal Diagnosis 32, 9961001.

Tsangaras, K., Siracusa, M. C., Nikolaidis, N., Ishida, Y., Cui, P., Vielgrader, H. \& Greenwood, A. D. (2014). Hybridization capture reveals evolution and conservation across the entire koala retrovirus genome. PLOS ONE 9, e95633.

Xiang, Y., Zhang, J., Li, Q., Zhou, X., Wang, T., Xu, M. \& Zhao, X. (2014). DNA methylome profiling of maternal peripheral blood and placentas reveal potential fetal DNA markers for noninvasive prenatal testing. Molecular Human Reproduction 20, 875-884.

Yin, A., Zhang, X., Wu, J., Du, L., He, T. \& Zhang, X. (2012). Screening significantly hypermethylated genes in fetal tissues compared with maternal blood using a methylated $\mathrm{CpG}$ island recovery assaybased microarray. BMC Medical Genomics 5, 26.

Zimmermann, B., ElSheikhah, A., Nicolaides, K., Holzgreve, W. \& Hahn, S. (2005). Optimized realtime quantitative PCR measurement of male fetal DNA in maternal plasma. Clinical Chemistry 51, 1598-1604. 